\title{
Sous le signe de la pluralité: l'écriture des femmes migrantes au Québec
}

\author{
Maïr Verthuy et Lucie Lequin
}

\section{Under the Sign of Plurality: The Writing of Migrant Women in Quebec $^{1}$}

Quebec "culture" must be situated in a historical context. A francophone population concerned about being engulfed in a flood of North American anglophones, constructed an identity with certain foundational myths of biological and linguistic homogeneity just as fictive as the "divine" mission that foregrounded their connection with pre-Revolutionary France. Today Quebec is in the process of revising or replacing these with other myths. With the demystifying effect of their "different" gaze, migrant women writers, doubly marginalized in the dominant discourse, have an important role to play in change. The category of marginal accorded their writing must not be permanent. It is a function of the specific situation in which they constitute a margin only in relation to the "centre" of a traditional homogeneous Quebec that assigns any "different" production to its periphery. The canon must be transformed now to take into account a new genre produced by these Quebec women. Earlier, Quebec absorbed culturally different groups into the francophone majority. Now, this homogeneity is questioned, difference valorized, a "multicultural" past sought out. This has been the case particularly since the passage of Bill 101 obliged immigrants to integrate into the francophone majority and eliminated the option of connecting with the anglophone community. The migrants too have changed, no longer Catholic Europeans, but members of visible minority groups, people with very different cultures and religions. The question of cultural affiliation is raised in a new complex way. In present day Quebec there is a choice: the French language as language may be positioned not just against English, but against Tamil, Korean, Chinese, Arabic, etc. Or WITH. In the second case, Quebec literature should never have been viewed as exclusively French, for there has always been a substantial body of writing in English and now there is a corpus developing in Native languages, Italian, Greek, 
etc. Our preliminary research has produced an inventory of 68 migrant women writers from a variety of countries. Their individual cultural heritages are diverse and complex. The "parole métèque" or outsider's speech is in fact a "parole métissé" or hybrid speech. Aware of having lost a country of origin, they recreate it by telling their stories, such nostalgia accompanied, however, by a desire to tame the new snow-bound country and to make their voices heard, by creating a space in which a unique plural identity might take shape. Analysis has focused on four writers so far who relate their wanderings, intellectual and familial: Régine Robin, Anne-Marie Alonzo, Nadine Ltaif and Mona Latif Ghattas. In their semiautobiographical narratives, they evoke the two countries as they interrogate exile and identity. In this study, we have chosen to focus on exile, breifly in the first three writers, centring our analysis on the work of Mona Latif Ghattas.

Qui dit culture québécoise dit une culture nécessairement dès sa naissance un peu en porte-à-faux, dans la mesure où la population francophone traditionnelle du Canada français avait besoin de se construire une identité contre cet océan nord-américain dans lequel elle risquait de se noyer. C'est dans ce contexte historique qu'il faut situer le développement par ce peuple d'un certain nombre de mythes fondateurs portant essentiellement tant sur une homogénéité biologique et linguistique toute fictive chez ses ancêtres que sur sa mission «divine», c'est à dire la nécessité en terre hostile de maintenir la religion catholique par le biais de la langue française ainsi que le souvenir d'une certaine culture française pré-révolutionnaire.

Aujourd'hui, pour des raisons autres, le Québec trouve nécessaire de réviser quelque peu ces anciens mythes, voire de les remplacer complètement. Il importe dans ces conditions de prêter une attention particulière à la voix des auteurs migrants et particulièrement à celle des migrantes, doublement marginalisées dans le discours séculaire, puisque leur apport permet d'une part de porter un regard différent sur le Québec actuel mais aussi peut-être de démystifier les nouveaux mythes en train de s'élaborer.

Il serait souhaitable aussi ce faisant de ne pas accorder à ces écrits un statut spécifique ou marginal permanent. Si dans un premier temps un tel classement s'impose, celui-ci doit par la suite disparaître, car le maintenir serait accepter que se perpétue cette vision traditionnelle et dépassée du Québec selon laquelle le centre composée de «francophones 
de souche» est un et indivis. Dans cette optique, seule l'existence nouvelle d'une périphérie - à laquelle l'on renvoie quand-même toute production «différente» - permettrait de distinguer entre hier et aujourd'hui. Le canon lui-même doit maintenant se transformer pour tenir compte de l'apport de ces Québécois-es d'un genre «nouveau.»

Il conviendrait peut-être pour mieux faire comprendre l'évolution de cette société d'y voir une espèce de succession. Après la Conquête et ses suites, une population francophone catholique s'est retrouvée dans une situation de faiblesse par rapport au Canada anglais. Largement repliée sur elle-même, elle a produit sa propre élite de clercs destinés à devenir les mandarins, la nomenclature, du Québec. La province est devenue leur fief à l'intérieur de ce continent; ce sont ces gens qui ont exercé le pouvoir décisionnel dans de nombreux domaines (éducation, politique linguistique et religieuse, vie artistique et culturelle, etc.) et ont ainsi donné forme à l'identité québécoise.

Ici et là ont pu se rajouter au groupe francophone un Irlandais ou deux ${ }^{2}$, un ou plusieurs Écossais, quelques Allemands, des Juifs convertis (ou marranes) et plus récemment des Italiens, des catholiques donc pour la plupart. Tous ces gens ont été occultés dans l'ensemble, absorbés dans la population québécoise majoritaire, renonçant par là aux origines.

Mais là où auparavant, l'on pouvait exiger que toute différence s'estompe, que le nouveau venu ou la nouvelle venue se fonde dans la population générale, que la prétendue homogénéité ne soit pas mise en cause, $l^{\prime}$ 'on a tendance aujourd'hui à faire valoir cette présence autre car dorénavant le Québec doit faire peau neuve, se chercher une nouvelle identité. Il a besoin aujourd'hui de se trouver ou retrouver un passé «multiculturel». Il est probable que cela ne se passe pas autrement dans tous les pays à tradition européenne mais le changement se présente de façon aiguë au Québec depuis la loi 101, loi votée dans les années 70, et qui, pour la première fois, mettait les immigré-e-s dans l'obligation de s'intégrer à la majorité francophone.

Avant cette date, les immigré-e-s pouvaient choisir entre celle-là et la communauté anglophone, la plupart choisissant cette dernière pour diverses raisons. Aujourd'hui ces personnes sont donc obligées d'aller vers le Québec francophone. Il ne s'agit plus alors d'absorber un «étranger» ici et là, mais de faire de la place à des gens très variés qui arrivent par milliers. Alors les «vieux» Québécois, composés de gens de souche européenne, même s'ils sont prêts à ne plus se reconnaître 
comme étant entièrement de souche française - et catholique - se trouvent confrontés à un nouveau choix, parfois douloureux: s'ouvrir ou non à des personnes - nombreuses - qui appartiennent souvent à des minorités visibles et/ou à des cultures très différentes - hindoue, musulmane, etc. De toute manière, l'immigré-e moderne ne s'assimile plus, il ou elle s'intègre certes, mais avec sa différence; on peut chercher à maintenir sa culture d'origine dans son pays d'accueil, de toute manière l'on maintient des contacts avec la culture que l'on a quittée, l'on retourne dans son pays, y envoie ses enfants et ainsi de suite. Dans certains cas, l'immigré-e n'a aucun statut juridique; dans d'autres, cette personne possède jusqu'à trois autres nationalités. La question de l'appartenance se pose de façon tout à fait nouvelle.

Deux problèmes au moins se posent, dont le moindre n'est pas pour le Québec la nécessité de se constituer un passé d'accueil, soit, par exemple, pour mieux préparer les Québécois d'aujourd'hui à accueillir effectivement cette nouvelle population, ou pour convaincre les arrivant-e-s qu'ils et elles sont effectivement les bienvenu-e-s au Québec et ne doivent pas craindre des changements constitutionnels éventuels.

Le deuxième problème viendrait de la nécessité concomitante de comprendre et accepter que les citoyen-ne-s du Québec aient deux ou plusieurs patries, avec tout ce que cela entraîne au niveau identitaire. Dans l'ensemble, les nouveaux ou nouvelles arrivé-e-s ne peuvent pas n'être que Québécois-es. Leur vision est autre. Aimer le Québec certes, mais garder une ouverture sur l'Inde ou la Nouvelle-Guinée ou l'Angleterre même. Le Québec aujourd'hui doit alors aussi comprendre que le français comme langue ne se joue plus contre l'anglais, ou plus seulement contre l'anglais, mais contre le tamoul, le coréen, le chinois, l'arabe, etc. Ou AVEC. Voilà le choix. La littérature québécoise n'aurait jamais dû être perçue comme uniquement francophone puisque de tout temps il a existé une importante littérature en anglais; aujourd'hui elle ne peut plus l'être, car, à côté des "migrants-e-s» qui écrivent effectivement en français, il se développe tout un corpus dans diverses langues, autochtones, italienne, grecque, j'en passe.

Voilà le contexte nouveau dans lequel se situe notre travail.

Notre recherche sur l'écriture migrante des femmes n'en est qu'à ses débuts. Dans un premier temps, nous avons répertoriéla production des femmes migrantes de 1960 à 1991. Cet inventaire compte soixante-huit écrivaines. Il reste encore quelques noms à confirmer. La moitié des écrivaines de notre corpus sont originaires de France ou de Belgique; 
quelques-unes sont nées au Québec de parents immigrés ou à ascendance culturelle diversifiée (un parent immigré et un parent québécois de souche; un parent blanc et un parent amérindien ou encore deux parents immigrés d'origine différente) ${ }^{3}$. Plusieurs auteures viennent $\mathrm{d}^{\prime}$ Europe de l'Est. Depuis les années quatre-vingt, les auteures viennent de plus en plus des pays du Sud: les pays arabes, l'Amérique du Sud, les Caraibes. Enfin quelques auteures originaires de pays anglophones ont choisi d'écrire en français. Le français est donc pour la moitié des auteures une langue seconde ou encore une langue imposée à leurs ancêtres, à leur pays.

Ultérieurement, il faudra analyser ces variantes de langue, de pays d'origine, de période de contact avec le pays hôte, du parcours esthétique particulier à chaque auteure.

Nous avons commencé à analyser l'importance de la culture d'origine, collective et/ou familiale dans le regard porté sur la culture québécoise. A ce stade-ci de notre recherche, nous pouvons déjà noter la forte parenté entre les auteures issues de culture déjà fortement métissée, plurielle et/ou de famille où les migrations, l'errance et les mélanges ethniques ont fibré la généalogie familiale. Ces dernières observent avec étonnement, réserve et parfois douleur, le mythe de la culture québécoise monolithique ou le mythe du Québécois de souche. Simultanément, elles notent la pluralité que souvent l'on ne voit pas et souhaitent la prise de parole plurielle, la cohabitation et l'enchevêtrement de voix différentes. Conscientes de la perte du pays d'origine, elles le racontent et le recrée, mais cette nostalgie accompagne leur désir d'apprivoiser le pays de neige et d'y faire entendre leur voix, $\mathrm{d}^{\prime} \mathrm{y}$ dessiner un espace où une identité singulière et plurielle pourra prendre forme.

Nous avons débuté l'analyse de la pluralité par l'étude de quatre auteures, trois originaires d'Égypte et une de France: Régine Robin, Anne-Marie Alonzo, Nadine Ltaif et Mona Latif Ghattas. Il serait cependant trop long de parler de chacune des auteures, pourtant de proche parentes non seulement par leur errance, leur façon d'aborder l'exil, mais surtout parce que l'exil subi est aussi recherché pour son pouvoir d'influence dans leur création, dans leur vie et dans la transformation de la culture et société québécoises. Pour continuer de vivre dans l'exil, ces quatres auteures, quatre intellectuelles, racontent leur passé, leur famille, leur culture. Leur généalogie se rapproche par le métissage, l'errance, les interférences culturelles, la pluralité de langues et de reli- 
gions dans leur vie ou dans leur milieu. Sans être des autobiographies, plusieurs de leurs oeuvres comportent des éléments autobiographiques; le «je» de la narration est parfois, souvent, le «je» autobiographique. Les deux réalités culturelles, celle du pays d'origine et celle du pays d'accueil, sont présentes, mais de façon plus ou moins marquée. Sous le signe de la pluralité, elles interrogent l'exil et l'identitaire qui peut s'y former. Nous avons choisi de parler surtout de l'exil, muse et créateur, de Mona Latif Ghattas.

Mais avant quelques lignes sur l'exil des autres. C'est très tôt que la narratrice d'Alonzo se découvre autre, car ce «je» est peu Égyptienne étant donné sa généalogie «si peu arabe» $(\mathrm{D}, 5)$, plusieurs fois métissée, depuis bien des générations: «toujours étrangère marquée là-bas comme ici» $(D, 5)$ L'appartenance ne joue nulle part: «Au fond en vérité de pays aucun» $(\mathrm{B}, 33)$. Quant à Nadine Ltaif, son exil est «celui qui prend racine aux racines du déracinement» $(E, 28)$. C'est donc dans un espace de schizophrénie $(E, 48)$ que l'exilée de Ltaif habite, ce territoire entre deux réalités; elle ne voudrait cependant ni se percevoir comme le produit d'une seule histoire ni éprouver une appartenance culturelle et historique fixe qui la perdait. Cet exil craint et recherché, devient «le souffle interrompu, et la traversée renouvelée de l'éternelle histoire.» (E, 51) Chez Régine Robin, le temps de l'entre domine aussi: «Désormais le temps d'ailleurs, de l'entre-trois langues, de l'entre-deux alphabets, de l'entre-deux mers, de l'entre-deux mondes, l'entre-deux logiques, l'entre-deux nostalgies» $(\mathrm{Q}, 67)$ dit la narratrice de La Québécoite. Cette exilée française et juive est aussi de nulle part et se découvre autre dans le même.

Mona Latif Ghattas, née au Caire en 1946, vit au Québec depuis 1966. Écrivaine, elle a aussi signé plusieurs mises en scène et des extraits de ses oeuvres écrites ont été le point de départ de spectacles à Montréal, à Québec et en 1990, en Belgique.

Dans l'espace blanc du Québec que le destin lui a réservé, elle a apprivoisél'espace de l'écriture. Avec le recul que lui accordent le temps et l'exil, elle peut chanter le monde de son enfance. Dans ses premières oeuvres, elle donne son Orient au lectorat du pays des neiges, mais toujours elle se situe dans l'espace de l'exil. Dans son dernier roman, Montréal devient l'univers imaginaire investi et l'Orient, un désert d'Anatolie, se manifeste en différé par les contes d'un sans-papiers, d'un réfugié clandestin à Montréal. Il lui a fallu, dit l'auteure, vingt-quatre ans, pour intégrer l'Occident en elle. (Royer, 1990) 
Dans son premier roman Nicolas le fils $d u$ Nil, elle raconte l'histoire de son père à partir de l'exil du grand-père, un Syrien venu tenter sa chance en Égypte où il a choisi une femme et le Nil. Ce roman poème, écrit en exil afin de pouvoir continuer à vivre, de pouvoir avancer, annonce les oeuvres à venir de Latif Ghattas, l'exil qu'elle doit dire, l'Orient qu'elle doit donner, les femmes qu'elle veut chanter.

D'oeuvre en oeuvre, plaçant son coeur à l'état oriental, elle se fait scribe pour transmettre à son fils né dans la neige, son héritage culturel, mais aussi et surtout pour transcrire la mémoire des odeurs, des objets et des chants anciens entendus dans l'enfance afin de comprendre l'Orient blessé et le désarroi du monde. Chaque oeuvre entremêle un plus la géographie: le Saint-Laurent commence à côtoyer le Nil dès le deuxième recueil. Devant la page de neige, elle s'affirme femme, poète, Égyptienne et Québécoise, car dans son jardin de neige, elle peut continuer d'écouter les anciennes tempêtes de sable.

Dans chaque oeuvre, elle fait entendre une multiplicité de voix de femmes récitant la souffrance, l'audace et/ou les transgressions, en dehors du discours revendicatif, dans l'action du quotidien. Set El Kol (Les voix du jour et de la nuit), son personnage féminin le plus fort, naît et renaît des racontars de femmes qui prennent ainsi un pouvoir réel de subversion. C'est une oeuvre qui participe de l'impossibilité de raconter à une voix, de ne raconter qu'une fois. Dans son dernier roman Le double conte de l'exil, elle fait entendre une voix de femme et une voix d'homme, mais chacune contient plusieurs autres voix, des voix de femmes surtout. Deux douleurs se rencontrent; l'une, Amérindienne, en exil dans son propre pays, l'autre, un réfugié, balafré par la guerre dans un désert d'Anatolie. Ils n'ont d'identité que leur déracinement. Mais la voix de Mariam Nour issue de l'homme rapproche la femme, Madeleine/Manitakawa, de son identité de femme et d'Amérindienne; elle se remet à vibrer et Fêve, au contact de la femme, entre dans le présent, dans la vie. Ils n'arriveront cependant pas à vivre à Montréal; rejeté par l'immigration, Fêve est expulsé; Manitakawa rentre dans son village, incapable de vivre avec les Blancs.

L'intériorisation de l'exil sous-tend l'écriture de Mona Latif Ghattas qui, peu à peu, laisse circuler la différence/les différences. «[Elle] interprète les signes. Et les signes parlent pour toutes les langues.» $(\mathrm{QV}, 54)$ Dans le prologue de son dernier roman Le double conte de l'exil, Mona Latif Ghattas annonce un double récit de survivance aux «blessures de l'histoire» et au «chantage de l'histoire» ( D, 10). Cette douleur, nous dit 
la narratrice, ne meurt jamais. Cependant, «l'étreinte accueillant à la fois le même et l'autre parvient miraculeusement à cautériser le mal vivant» (D, 11). C'est dans l'exploration et l'acceptation du métissage culturel, élément fibrant la généalogie familiale avant même la naissance de son père, à l'écoute de la mémoire des odeurs et des objets, mémoire et parfois avenir racontés surtout par une multitude de voix de femmes que Mona Latif Ghattas forge au jour le jour son identité de femme et d'écrivaine, refusant l'étroitesse d'une identité unique, sans mouvement.

Si l'on peut émettre le voeu que ces écrits de l'autre appartenance évitent de figer dans un passé mort l'image de l'ancien pays ou del'autre culture, ce qui est le cas de nos auteures, il faut néamoins que tous les écrits trouvent leur place, trouvent un accueil. Cette littérature assez nouvelle à l'intérieur de nos frontières exige un effort certain de la part des lecteurs-lectrices ainsi que de la critique. Les écrits aussi peuvent jouir d'une double - ou triple - appartenance: faire partie de la diaspora haïtienne, par exemple - et ce n'est qu'un exemple - tout en adhérant à une certaine québécitude réinventée. Pour nous, au Québec, le meilleur moyen non seulement de nous construire un avenir à l'abri du racisme mais aussi de développer une littérature encore plus riche, c'est justement de veiller à ce que la culture majoritaire ne soit plus présentée comme telle; que l'on présente une culture, des cultures, éclatées, diversifiées, chacune apportant sa contribution à l'élaboration d'une nouvelle identité québécoise mouvante. Collective, mais mouvante. Nous souhaitons que cette recherche contribue à cette entreprise commune.

\section{Notes}

1. Nous avons retenu le terme migrante car nous voulons surtout étudier les interférences et les appartenances culturelles. Plus large que le terme immigrée, migrante permet $\mathrm{d}$ 'inclure les auteures de la première génération nées au Québec et les Amérindiennes; il suggère le mouvement, la dérive. Nous souhaitons rappeler que ce texte est une version remaniée d'une communication présentée au colloque Création et réalité d'expression française organisé par la Société des professeurs français et francophones d'Amérique, tenu à New York en mars 1992.

2. Ici le masculin comprend effectivement le féminin.

3. C'est le cas de Marie-Françoise Taggart, de Lisa Carducci, de Désirée Szucsany, etc. 


\section{Ouvrages Cités}

Afin de ne pas multiplier les notes en bas de pages, les titres des auteures migrantes sont accompagnés d'un code entre parenthèses qui sera inséré dans le texte suivi de la page citée.

Alonzo, Anne-Marie. Droite et de profil. Montréal: Lèvres urbaines no 7 , 1984, 14 p. (D)

—. Bleus de mine. Saint-Lambert: Éditions du Noroît, 1985, 68 p., ill. (B)

—. Écoute, Sultane. Montréal: Éditions de l'Hexagone, 1987, 136 p. (É)

—. Esmaï. avec une traduction en hiéroglyphes de Nihal Mazloum. Montréal: Nouvelle Barre du Jour, 1987, 45 p. (ES)

- La vitesse du regard, Autour de quatre tableaux de Louise Robert. Laval: Trois, 1990, 39 p. (V)

Latif Ghattas, Mona. Nicolas le fils du Nil. Égypte: Elias Modern Publishing House \& Co., 1985, 158 p. (N)

- Quarante voiles pour un exil: récits et fragments poétiques. Laval: Éditions Trois, 1986, 105 p. (QV)

—. Les voix du jour et de la nuit. Montréal: Boréal, 1988, 119 p. (V) ).

—. Le double conte de l'exil. Montréal: Boréal, 1990, 171 p. (D)

Ltaif, Nadine. Les métamorphoses d'Ishtar. Montréal: Guernica, 1987, 70 p. (M)

—. Entre les fleuves. Montréal: Guernica, 1991, 51 p. (E)

Robin, Régine. Le cheval blanc de Lénine ou l'histoire autre. Bruxelles: Éditions Complexes, 1979, 150 p. (C)

—.La Québécoite. Montréal: Québec/Amérique, 1983, 200 p. (Q)

Royer, Jean. «Mona Latif Ghattas est passée de l'exil à l'écriture». Le Devoir. 26 mai 1990, p. D-1. 